\title{
Relatos de vida de estudiantes adolescentes en contexto de contradicción cultural al interior del aula*
}

\section{Life Stories of Teenage Students in the Context of Cultural Contradiction within the Classroom}

Recepción: 09 Julio 2015 | Aprobación: 22 Febrero 2018

\author{
Marcela Suckel Gajardo \\ Universidad de Concepción, Chile \\ Daniela Campos SaAvedra ${ }^{\mathrm{a}}$ \\ Universidad de Concepción, Chile \\ ORCID: http://orcid.org/0000-0001-7818-6608 \\ Abelardo Castro Hidalgo \\ Universidad de Concepción, Chile \\ Cecilia Maldonado Elevancini \\ Universidad de Concepción, Chile \\ Laura SaAvedra Burgos \\ Universidad de Concepción, Chile
}

a Autor de correspondencia. Correo electrónico: dancampos@udec.cl

Para citar este artículo: Suckel Gajardo, M., Campos Saavedra, D., Castro Hidalgo, A., Maldonado Elevancini, C., \& Saavedra Burgos, L. (2018). Relatos de vida de estudiantes adolescentes en contexto de contradicción cultural al interior del aula. Universitas Psychologica, 17(2), 1-11. https://doi.org/10.111 44/ Javeriana.upsy17-2.rvea

\section{RESUMEN}

El objetivo de este trabajo fue analizar las vivencias de adolescentes con respecto a su paso por la escuela, caracterizando la contradicción cultural al interior del aula en contextos de vulnerabilidad social y pobreza económica. Desde un enfoque biográfico se entrevistaron 42 estudiantes de octavo año básico de siete escuelas de la provincia de Concepción (Chile). Los resultados muestran características de un sentido de identidad cultural y de diferenciación respecto del rol y la posición del estudiante en la escuela, la cultura escolar y la forma en que se expresa en el aula, a través de sus profesores. Para algunos estudiantes la escuela es complementaria a "proyectos de vida". Para otros, la cultura de la escuela representa el lugar donde no se sienten libres, generando interpretaciones contradictorias a su identidad cultural.

Palabras clave

relatos de vida; proyecto de vida; complementariedad y contradicción cultural; vulnerabilidad.

\section{ABSTRACT}

The objective of this work was to analyze the experiences of adolescent students with respect to their schooling by characterizing the cultural contradiction within the classroom in those that operate in the contexts of social vulnerability and economic poverty. 42 eighth-grade students from seven educational establishments from the area of Concepción, Chile, were interviewed with a focus on utilizing the biographical method. The results show characteristic features of a sense of cultural identity, as well as a sense of differentiation regarding the role and position of the student at the school, the culture which that position represents, and how it manifests itself in the classroom via the teachers. For some students, the 
school as complementary to "life projects". For other students, school represents some misinterpretations with respect to their cultural identity.

Keywords

life stories; life projects; cultural complementarity and contradiction; vulnerability.

En el escenario de contradicción cultural al que se ven enfrentados los estudiantes con una herencia cultural propia que en una primera mirada se contradice con la cultura escolar, resulta de interés indagar en las relaciones explícitas e implícitas que se dan entre profesores y estudiantes y entre estudiantes, con el propósito de describir y caracterizar las relaciones descritas al interior del aula en escuelas que funcionan en contextos de vulnerabilidad social y pobreza económica, especialmente, cuando no es considerada la herencia cultural y habitus de los estudiantes como parte importante del quehacer educativo del sistema escolar.

Estudios relacionados con relatos de vida de los escolares en América Latina dan cuenta de que los estudiantes, conscientes de las discriminaciones fundadas en la carencia de un capital económico, social y simbólico, intentan resistir la exclusión, integrándose en relaciones de subordinación y dominación. La resistencia en este registro de exclusión no se juega en apuestas para cambiar el orden, sino para tener un lugar dentro del mismo (Brandi, Filippa, \& Schiattino, 2009). A este respecto, la relación que algunos estudiantes establecen con la escuela se presenta matizada por la percepción de que esta es un obstáculo que deben soslayar, transformando su paso por ella en la única vía para salir adelante.

Las teorías de la reproducción social de Bernstein (1990), Bourdieu y Passeron (1977) y Willis (1988) se aproximan a este fenómeno, declarando las diferencias de rendimiento escolar como producto de un "déficit". Esta explicación está dada por la relación que se establece entre dos culturas distintas y su encuentro en la escuela (Castro, Figueroa, \& Maldonado, 2012), el cual puede establecerse en condiciones de contradicción, cuando la cultura de los estudiantes es desplazada.
De acuerdo con Bernstein (1977), los códigos elaborados son los que predominan en la escuela, por consiguiente, los estudiantes que utilizan códigos restringidos quedan en desventaja (Castro et al., 2012). De este modo, los códigos lingüísticos que portan los estudiantes constituyen un aspecto de identificación social en el campo educativo, que puede llegar a configurar un factor de discriminación en la medida en que estos no sean aceptados ni valorados en su diversidad.

Según Bourdieu y Passeron (1977), la cultura dominante se expresa en el currículum de manera neutral, por tanto, la cultura de los trabajadores no es reconocida por este, siendo desplazada de la escuela. El encuentro entre estas dos culturas se refleja en habitus distintos y muy arraigados en los estudiantes, según el estrato social al que pertenecen. Por esta razón, la contradicción cultural para los estudiantes en condición de vulnerabilidad y pobreza socioeconómica es mayor que la que pueden llegar a experimentar los estudiantes de estratos sociales de clase media y alta (Castro et al., 2012).

De acuerdo con Willis (1988), en la reproducción social de la cultura escolar formal como cultura dominante es posible encontrar dos grupos, cuyas reacciones difieren, principalmente, en la manera de enfrentarlo este proceso de dominación. El primero está conformado por aquellos que optaron por ceder de manera silenciosa a esta nueva reacomodación cultural, quienes no solo legitiman la cultura impuesta, sino que, además, desean que la manifestación de cualquier tipo de resistencia sea corregida. El segundo grupo manifiesta su resistencia ante la cultura dominante, y se caracteriza por presentar un resentimiento reprimido que no alcanza a manifestarse en una confrontación abierta, sino que crea sus propios códigos, normas, conductas y relaciones, configurando una cultura contraescolar.

$\mathrm{Si}$ bien estas teorías abordan desde una perspectiva explicativa el fenómeno de la reproducción social, no ofrecen la posibilidad de superar la contradicción cultural presente en la escuela. Si se asume que el rendimiento escolar por estrato social es un fenómeno sociológico, 
sobre el cual la escuela pareciera no tener incidencia, entonces la consecuencia directa de dicha afirmación es que esta queda sin capacidad de dar respuesta a la contradicción cultural planteada. La realidad muestra que hay alumnos que sí logran superar la contradicción, precisamente, en las escuelas donde esta debiera expresarse en su forma más aguda, es decir, aquellas situadas en los lugares suburbanos más pobres.

Desde la perspectiva de la dialéctica de los aprendizajes, Castro et al. (2009) señala que superar la contradicción cultural es posible a partir de formas de trabajo pedagógico que permitan acceder a un nivel superior de abstracción en las relaciones didácticas al interior del aula. Existe una relación entre lo aprendido y lo por aprender que puede ser complementaria, contradictoria o contradictoria antagónica, relaciones que permiten explicar los procesos de interacción y enseñanza-aprendizaje en el aula, donde el encuentro de culturas opuestas se complementan o tensionan la escuela. La facilidad o dificultad con que se aprenda algo nuevo dependerá de esta relación, por tanto es posible pensar que la relación entre los estudiantes que resisten la escuela y la cultura escolar no es antagónica por naturaleza, sino que se transforma en tal, cuando no se valora e incorpora al trabajo escolar, la visión de la praxis que esos estudiantes traen consigo (Castro, 2009; Castro et al., 2012).

En los sistemas educativos, en sus actividades centrales expresadas en los procesos de enseñanza-aprendizaje, confluyen diversas culturas: por un lado, la cultura de la comunidad educativa, de los profesores y de la sociedad en general y, por otro, la de los estudiantes y sus familias (Bourdieu \& Passeron, 1977. En este encuentro de culturas, existen dos actores principales: el profesor quien, teóricamente, debe desarrollar un currículum y el estudiante que, desde su formación particular, asume el aprendizaje del mismo. Ambos en distinto grado traen consigo distintos códigos, los cuales adquieren especial relevancia en el rendimiento escolar de los estudiantes, ya que para ellos los códigos culturales usados por el profesor pueden resultar ajenos a su cultura y, por tanto, disminuir su motivación por aprender lo que la escuela espera de ellos (Zapata, Castro, \& Rodríguez, 2013).

La dialéctica de los aprendizajes considera la distancia entre la cultura escolar formal y la cultura de los estudiantes, dando al profesor la posibilidad de superar esta brecha al establecer puentes didácticos entre ambas culturas, al constituir estos una guía para la acción. Esta distancia no se puede comprender sin considerar los relatos de vida de los estudiantes, cuando ellos aún no han terminado la escuela y pueden dar cuenta como protagonistas. Por tal motivo, la aproximación a esta problemática se aborda desde la perspectiva de los estudiantes respecto de su trayectoria escolar en curso.

En este contexto, se aborda la siguiente interrogante: ¿Cuáles son las tensiones $\mathrm{O}$ contradicciones culturales entre profesores y estudiantes y entre estudiantes que se dan al interior del aula, en escuelas insertas en contextos de vulnerabilidad social?

Desde una aproximación cualitativa y biográfica fue posible acceder a los relatos de vida de estudiantes adolescentes, con el objetivo de describir y analizar las percepciones de los estudiantes con respecto a su paso por la escuela.

\section{Método}

El método que sustenta esta investigación es el enfoque biográfico, en específico, la utilización de relatos de vida. Este enfoque permite la utilización del relato de vida bajo un determinado marco conceptual, ético y epistemológico. En la dimensión conceptual, el relato configura definiciones y diferenciaciones con los demás, cumpliendo una función en la construcción identitaria que permite dar sentido a las acciones o a los eventos vividos. En su dimensión ética, el relato de vida implica una opción ineludible: la de tomar posición con respecto a lo que cuenta (Cornejo, Mendoza, \& Rojas, 2008). En su dimensión epistemológica, se establece una relación sujeto-sujeto, en la que "el narrador no es el único que habla, piensa y se 
transforma" (Bertaux, 1993 citado en Cornejo et al., 2008, p. 31), sino que también el oyente o narratario realiza un aporte desde su posición.

La elección del enfoque biográfico se fundamentó en la coherencia que se establece entre este y la problemática en estudio, toda vez que constituye un enfoque teórico metodológico que permite conciliar lo social y lo psicológico, rescatando la subjetividad de la narración como fuente de conocimiento, así como una forma de expresión y construcción de la identidad (Bertaux, 1999; Cornejo, 2006; Cornejo et al., 2008).

\section{Participantes}

Los participantes del estudio constituyen 42 estudiantes de 8vo. año básico de siete escuelas municipalizadas de la provincia de Concepción (Chile), ubicados en sectores de alta vulnerabilidad y pobreza económica.

Para la elección de los participantes se establecieron principalmente dos criterios: (a) ser estudiante de octavo año básico y (b) asistir a una escuela ubicada en un sector de alta vulnerabilidad y pobreza económica. El primer criterio implica afrontar la finalización de un ciclo de enseñanza que permite a los participantes reflexionar en torno a su trayectoria escolar, desde una mirada retrospectiva del proceso educativo. El segundo criterio es una condición que aumenta las posibilidades de que emerja un conflicto entre la cultura escolar y la cultura del estudiantado.

\section{Diseño}

En la recolección de los relatos de vida, se realizaron dos encuentros con los participantes, con una duración promedio de una hora y media cada uno. En el primero de ellos, los participantes escribieron un relato de vida de sus experiencias asociadas en la escuela y en el segundo encuentro realizaron un relato oral de dichas experiencias. Ambos encuentros se llevaron a cabo en la escuela a la que asisten los participantes, en salas facilitadas por cada establecimiento y en un horario acordado con cada uno de ellos. Los intervalos entre el primer y segundo encuentro fluctuaron entre uno y tres meses. Los relatos orales fueron grabados en su totalidad, previo consentimiento explícito por parte de los participantes. La transcripción fue realizada sin editar, intentando ser lo más fiel posible con el relato.

\section{Procedimiento}

Para la producción de los relatos de vida se realizó un primer encuentro con los participantes, durante el cual fueron invitados a conversar acerca de sus vivencias a lo largo de su paso por la escuela. No se restringió experiencia escolar alguna, se respondieron consultas y se comunicó el marco de confidencialidad en el que se enmarca el estudio. En esta instancia, se los instó a plasmar las vivencias que recuerdan por escrito, utilizando para ello la consigna "Cuéntanos cómo ha sido tu paso por la escuela, lo que tú quieras contarnos de la escuela...". Una vez que han finalizado, se les consultó la posibilidad de ser entrevistados para efectos de profundizar en aspectos de su trayectoria de vida escolar, plasmados en sus relatos en formato escrito. Es así como se concretó el segundo encuentro, durante el cual cada uno de los participantes volvió sobre su relato escrito, complementándolo con una narración oral, profundizando en diferentes aspectos planteados por ellos relacionados con la mayor o menor tensión con la cultura escolar.

\section{Análisis de datos}

Con relación al enfoque biográfico, diversos autores plantean que no existe un método único para el análisis de los datos (Lainé, 1998; Legrand, 1993 en Cornejo et al., 2008, p. 37), sino más bien, este análisis se define en consideración a los objetivos de la investigación, al fenómeno estudiado y a las consideraciones epistemológicas y metodológicas con respecto a la construcción de conocimiento científico, planteando una diversidad de posibilidades (Cornejo, 2006; Cornejo et al., 2008). 
Desde el enfoque biográfico y considerando del objetivo de este estudio, resulta fundamental acceder a la comprensión de los estudiantes acerca de sus propias vivencias, constituyendo este insumo el punto de partida para categorizar y analizar los relatos, a la luz de las relaciones dialécticas complementarias, contradictorias o contradictorias antagónicas que se establecen entre lo aprendido y lo por aprender, en el marco de las trayectorias de vida escolar de los participantes y en el contexto de contradicción cultural al interior del aula.

El análisis de los relatos se desarrolló en tres etapas en las que participó un equipo de cinco investigadores expertos en la temática. En la primera, se procedió a la lectura colectiva de los relatos escritos de cada participante por parte del equipo de investigadores. Esta lectura no incluyó los datos de identificación del sujeto participante ni de la escuela de procedencia. A partir de esta lectura, se destacaron los elementos característicos que permiten describir la relación dialéctica del participante con la escuela. En la segunda etapa, se buscó entender las diferencias, semejanzas y particularidades de cada relato, en función de las relaciones dialécticas construidas por los sujetos. A partir de este análisis, emergen las siguientes categorías: (1) la vida escolar como parte de un proyecto de vida, (2) la vida en la escuela cuando esta resulta ajena y contradictoria y (3) la vida escolar desde la contradicción y el antagonismo. En una tercera etapa, se analizaron los relatos orales en función de las categorías citadas anteriormente. En esta última etapa, se cauteló la relación de tensión entre los elementos teóricos y empíricos que emergen a partir de los relatos y el análisis de estos en función de las relaciones dialécticas construidas por los sujetos en el marco de las experiencias de vida escolar.

\section{Resultados}

El presente estudio pretendió indagar en las vivencias de los estudiantes respecto a su paso por la escuela en contexto de contradicción cultural, cómo se relacionan con esta y cómo interpretan su experiencia escolar. Para inquirir y comprender estas vivencias, el análisis de sus relatos de vida permitió categorizarlos en relaciones complementaria, contradictoria o contradictoria antagónica con la escuela. Mediante criterios de identidad y diferenciación entre los grupos de pares, fue posible acceder a las representaciones sociales de los estudiantes respecto de su relación con la escuela y sus profesores, desde lo académico, y de la dimensión personal implicada en la construcción de identidades en los estudiantes entrevistados.

\section{La vida escolar como parte de un proyecto de vida}

Se trata de relatos de vida donde la escuela es vista como algo natural y complementaria con una suerte de proyecto de vida difuso, pero con una meta de superación. Estos relatos consideran las narraciones provistas de experiencias de significancia con la cultura escolar que los estudiantes de 8 vo. año básico realizan respecto de su vida escolar, representada a través de la escuela como institución o de sus profesores/ as. La complementariedad con la escuela que estos estudiantes manifiestan se enmarca en el reconocimiento que hacen de esta, como un paso que deben sortear para surgir en la vida. A este respecto, esta complementariedad se refleja en dos aspectos fundamentales: (a) la valoración del contenido que la escuela les presenta y (b) la relación que establecen con los profesores.

En relación con el contenido de la escuela, es posible constatar la valoración que hacen los estudiantes de lo que aprenden y de cómo estos aprendizajes serán de utilidad en el mediano plazo y en términos de su proyecto de vida. Esta permite que los estudiantes le otorguen un sentido al contenido de la escuela y/o valor de uso al mismo.

[...] porque la materia que pasan me va a servir más adelante. (Mario, Escuela F) ${ }^{1}$

[...] todo me ha servido hasta el momento, todo lo que he aprendido me ha servido. (Diego, Escuela E)

Respecto a la relación profesor-estudiante y el proceso de valoración que los estudiantes hacen, 
es posible constatar una percepción favorable hacia los profesores/as que destinan tiempo de calidad y esfuerzos por reestructurar su enseñanza de acuerdo al capital cultural de origen de sus alumnos y a las posibilidades de construir aprendizajes a partir del habitus de los estudiantes.

[...] te explican bien la materia, si tienes alguna duda te la explican hasta que tú la entiendas [...] no, y hacen bien sus clases.

(Ana, Escuela B)

Con respecto a esta relación, además de la percepción favorable en lo que se refiere al profesor mediador, también emerge la figura de un profesor que no asume este rol y que se relaciona solo con el contenido que porta o puede portar el estudiante, es decir, reproduce.

[...] les hacía a los alumnos que les costaba más [...] se iba más a ellos, no como otros profesores que se van con algunos no más, que saben más.

(Juan, Escuela F)

[Es una buena profesora] porque [...] una, nos enseña bien; otra, nos ayuda en todo y otra siempre nos apoya en las decisiones que uno va a tomar [...] ella repasa y repasa lo mismo, o sea no es como los profesores que a veces no se le entiende y a esta sí se le entiende porque escribe en la pizarra, después pregunta si entendieron, después va explicando, después $[\ldots]$ alguna duda le preguntamos $[\ldots]$ y, es fácil.

(Diego, Escuela E)

Otro aspecto relevante con respecto a la relación profesor-estudiante, incluye la valoración de la persona del estudiante como un legítimo otro, sujeto de derechos. A partir de esta definición de relación, el proceso de interacción se fundamenta en un sentimiento de confianza que permite el desarrollo de habilidades cognitivas y socioafectivas de los estudiantes.

Siempre los profesores jefes [...] me han tocado profesores buenos [...] que enseña bien, además que a uno lo entiende, lo escuchan y siempre nos dicen que cuando tengamos problemas les contemos a ellos.

(Marcela, Escuela E)
Asimismo, los estudiantes perciben amenazada la relación que establecen con sus profesores/ as de manera complementaria, al sortear experiencias de fracaso académico.

Con los profes, bueno me llevo bien, igual que cuando con un profesor nos va mal, como que nos alejamos [...] si a uno le va mal es por nosotros, no tienen la culpa los profesores. (Andrea, Escuela C)

La percepción de una relación profesorestudiante amenazada por el desempeño académico que puedan lograr los alumnos se puede explicar a partir del desarrollo de relaciones fundadas en la adhesión a la cultura escolar como dominante y no necesariamente en la aceptación de un otro como legítimo otro. De esta manera, el proceso de identificación realizado por los estudiantes se aprecia como caracterizado por la proyección de la figura del profesor como profesional que estudia y se prepara para el desarrollo de su labor, situándolo en una posición idealizada desde la que se pueden relacionar si se subordinan o hacen propias las disposiciones culturales de sus profesores/ as (habitus) como representantes de la cultura dominante.

Bien, porque aquí en esta escuela, hay hartos profesores nuevos que saben más. (José, Escuela F)

Los alumnos que se relacionan de manera complementaria con la escuela, desarrollan estrategias asociadas al conocimiento y a la comunicación para sortear su paso por la institución escolar con éxito. Las estrategias de conocimiento permiten al estudiante elegir, coordinar y aplicar los procedimientos estimulados por los profesores para conseguir un fin relacionado con el aprendizaje acorde con las expectativas docentes, familiares y de sí mismos, a través de la escucha, la comunicación y la interacción, para lo cual despliega comportamientos adaptativos que le facilitan permanecer de manera óptima en la escuela y superar las contradicciones sociales y culturales en las que nació y se desarrolló (Zapata et al., 2013). 
La vida en la escuela cuando esta resulta ajena y contradictoria

La relación de contradicción se hace evidente, toda vez que el grupo de pares no logre dar con una interpretación que se acerque a sus disposiciones culturales o habitus. Los estudiantes que establecen esta forma de relación perciben la escuela como impositiva, con mandatos carentes de sentido, inaccesibles al entendimiento común y basados en una relación profesor-alumno/a, que los deja en una posición de vulnerabilidad respecto a la cultura dominante y restringe toda posibilidad de interactuar de manera autónoma con el medio o cultura escolar.

Es que a un profesor no le tengo tanta confianza como para contarle mis problemas.

(Miguel, Escuela C)

Con los más estudiosos no más, con los que se sientan adelante, a ellos les habla más.

(Ricardo, Escuela F)

Que los profesores nos entiendan más porque siempre les creen a los demás, no a nosotros $[\ldots]$ nunca $[\ldots]$.

(Matías, Escuela A)

El proceso de identificación en los estudiantes entrevistados da cuenta del establecimiento de relaciones de tipo contradictorio con la escuela y sus profesores a partir de un marcado sentido de pertenencia e identidad en torno al grupo de pares y por ende, desde lo colectivo. Con el grupo de pares comparten experiencias escolares de segregación que se presentan asociadas a sentimientos de exclusión y tensión escolar.

No me caen bien (los profesores) [...] no me hallo con ellos [...] te agarran y te echan pa' fuera y te suspenden [...] no hacen nada.

(Matías, Escuela A)

En el patio estoy como sin estrés, sin hacer nada, más libre $[\ldots]$.

(Miguel, Escuela C)

La actitud de los profesores, que algunos se preocupaban otros les daba lo mismo [...] siguen con la clase, todos callados y nadie habla [...] es como estresante eso, no dejan hablar y si uno tiene duda, queda con la duda no más.

(Gabriela, Escuela C)

Para este grupo de estudiantes la experiencia escolar adquiere significancia a partir del proceso de socialización con los pares, donde la interacción social pasa a ser el centro y agente motivador a través del cual se le hace más llevadero su paso por la escuela.

[...] cuando pienso que tengo que venir a estudiar, no $[\ldots]$ eso no me gusta, pero al momento de llegar aquí y encontrarme con mis compañeros ahí ya me empieza a gustar. [...] [Me gusta la escuela] porque aquí comparto con mis compañeros, nos divertimos, hablamos de las cosas que nos pasan.

(Camila, Escuela B)

[Me gusta la escuela] porque tengo hartos amigos, además que en la casa no tengo amigos $[\ldots]$ y allá no puedo tener amigos tampoco $[\ldots]$ porque en la población que vivo, pura maldad.

(Paulina, Escuela B)

Una vez me robaron una cosa $[\ldots]$ yo le quería pegar un combo al que me lo robó [...] mis amigos me dijeron que no porque estaba condicional en el colegio [...] me desahogué con ellos, hablé y después no le pegué, si no me podían echar del colegio [...].

(Matías, Escuela A)

En este escenario, los estudiantes contradictorios en su forma de relacionarse con la escuela, desarrollan estrategias que se circunscriben solo al plano de interacción social como una forma de permanecer y enfrentar las relaciones de contradicción cultural.

\section{La vida escolar desde la contradicción y el antagonismo}

Este grupo se caracteriza por presentar un comportamiento de resistencia a las normas que provienen de las figuras de autoridad en el contexto escolar, que se evidencia en el uso de violencia verbal explícita hacia compañeros/as y profesores y violencia no verbal principalmente hacia compañeros/as. 
[...] una pelea con un compañero. En el momento lo dejé inconsciente, entonces tomaron medidas y me echaron. Yo de chiquitito he sido maldadoso. O sea, yo pesqué un palo y lo agarré a palos.

(Alejandro, Escuela C)

Respecto al proceso de identidad desarrollado por los estudiantes entrevistados, es posible constatar experiencias de exclusión y rechazo de los pares hacia los otros, recurriendo a la violencia como patrón habitual de relación con el grupo curso o sus profesores. Asociados a las experiencias escolares de exclusión y rechazo, reportan sentimientos de soledad y frustración en el ámbito de las relaciones interpersonales con sus pares y de la relación profesor-estudiante.

Peleo a veces con un amigo y ando solo [...] a veces, nos molestamos a veces entre todos [...] no sé $[\ldots]$ no quiero andar con nadie $[. .$.$] estoy$ mal.

(Andrés, Escuela F)

Es el propio grupo de pares el que establece elementos de diferenciación con los estudiantes que establecen la relación contradictoria con la escuela de forma antagónica y explícita, mediante el uso de violencia verbal y/o no verbal.

[...] hoy en día, con los 'flaites' no se llega a nada [...] entonces uno me dijo oye 'vo cuico', te creí caballero.

(Alejandro, Escuela C)

Estos estudiantes no desarrollan estrategias sociales, de conocimiento o de comunicación que medien su paso por la escuela. Esto se explica por el nivel de contradicción que experimentan al interior de la institución escolar. Por consiguiente, es posible constatar en ellos un nivel de afectación emocional que impide un grado de sintonía afectiva con sus pares y profesores, una interferencia en la función ejecutiva y una distorsión en el proceso de toma de decisiones que les permita un comportamiento prosocial y la orientación al logro de metas de aprendizaje formales.

\section{Discusión}

A través de los relatos de vida de los estudiantes fue posible verificar aspectos característicos de un sentido de identidad y diferenciación respecto del rol y la posición de estos en la escuela. Dentro de estos aspectos, se encontraron diferencias en las visiones que reportan respecto de lo que significa para ellos la institución. Para los estudiantes complementarios con la cultura escolar, la escuela representa una posibilidad cierta de acceder a buen trabajo o continuar estudios. Para los estudiantes contradictorios, esta representa el lugar donde no se sienten libres, sino más bien conminados a estar bajo una serie de requerimientos con los cuales no compaginan y les generan interpretaciones contradictorias. Los alumnos que sortean su paso por la escuela con presencia de antagonismo en la relación de contradicción cultural, experimentan un grado de afectación en el ámbito emocional y cognitivo que implica una disminución en sus capacidades de establecer relaciones empáticas y tomar decisiones orientadas a la consecución de metas de aprendizaje formales.

Un aspecto de identidad que caracteriza al grupo de estudiantes que visualiza la escuela como un espacio y posibilidad cierta de obtener un trabajo, dice relación con la percepción de la figura del profesor/a como modelo susceptible de seguir o imitar. A este respecto, connotan de manera diferente a los profesores que logran motivarlos o que hacen esfuerzos didácticos en pos de su aprendizaje, de otros más tradicionales que centran sus esfuerzos en regular directamente la disciplina en la clase (Bellei, Valenzuela, Vanni, \& Contreras, 2014). No obstante, perciben amenazada la relación con el profesor, toda vez que el bajo rendimiento académico no sea congruente con la percepción de la relación, puesto que la dimensión cognitiva implicada resulta inconsistente con sus logros académicos. Esto puede explicarse a partir del desarrollo de relaciones fundadas en la adhesión a la cultura escolar como dominante y no necesariamente en la aceptación de un otro como legítimo otro.

Un aspecto de identidad para el grupo de estudiantes contradictorios dice relación con la 
percepción de la escuela como un espacio de interacción social con los pares, que les permite amortiguar las relaciones experimentadas al interior de ella como espacio educativo formal. En el escenario social descrito, este grupo genera un sentido de identidad en relación con el grupo de pertenencia.

Al considerar los aspectos de identidad y diferenciación asociados a la percepción de las normas y reglas en las escuelas, es posible constatar que los estudiantes en relación contradictoria se piensan y nombran desde lo colectivo. Para este grupo, son importantes las decisiones y las interpretaciones que puedan construir a partir del colectivo, por sobre lo individual. Esta situación marcaría un punto de inflexión en la relación profesor -estudiante durante el desarrollo de las clases, toda vez que el profesor/a se aleje de las posibilidades de ser interpretado de acuerdo al conocimiento que desde el grupo se genere y se exprese en el ámbito de la interacción.

Un aspecto diferenciador se visualiza en torno a la relación que los alumnos establecen con las normas y reglas. Para los estudiantes complementarios es relevante el establecimiento de normas y reglas que provengan desde fuera como lo sería una autoridad formal. En cambio, para aquellos con relación contradictoria, lo relevante es el establecimiento de normas y reglas de funcionamiento que provengan de un entendimiento colectivo y no impositivo de la autoridad por el solo hecho de pertenecer a la cultura escolar formal.

Por otra parte, los/as profesores/as que establecen relaciones con niveles de empatía adecuados y potencian la condición de persona en el plano de la interacción, son percibidos como accesibles y confiables. Cabe destacar que la construcción de este tipo de relación interpersonal se percibe amenazada tanto por estudiantes complementarios como contradictorios, en los casos en que el rendimiento académico no es congruente con la calidad de la relación interpersonal entre profesor/a y estudiante. No obstante, para aquellos con una relación contradictoria antagónica con la escuela, no se aprecia la progresión en los niveles de desarrollo de empatía en la relación profesor-alumno. Un aspecto que caracteriza a este grupo de estudiantes lo constituye el conocimiento que poseen respecto de la gradualidad de las normas y reglas que les son aplicadas, desde la autoridad formal, cada vez que las transgreden.

Otro aspecto diferenciador señala una relación con el uso de estrategias que los estudiantes movilizan o la carencia de ellas para sortear su tránsito por la escuela. Aquellos que establecen relaciones complementarias desarrollan estrategias asociadas al conocimiento y la comunicación, tanto para acceder a un aprendizaje acorde con las expectativas docentes como para desarrollar comportamientos adaptativos en congruencia con los requerimientos normativos de la escuela. Estas estrategias les superar las contradicciones socioculturales, habilitándolos para transitar por una cultura distinta a la propia, permanecer en la escuela y terminar el proceso con éxito (Zapata et al., 2013). Los alumnos con relaciones contradictorias desarrollan estrategias que se circunscriben solo al plano de la interacción social como una forma de permanecer y enfrentar las relaciones de contradicción cultural. Si bien estos estudiantes permanecen, la falta de estrategias de conocimiento los margina de la posibilidad de organizar su comportamiento en función de una meta de aprendizaje. Los alumnos que establecen relaciones antagónicas no desarrollan estrategias, lo que dificulta su permanencia en la escuela y agudiza la contradicción. Esto se expresa en un grado mayor de afectación emocional que dificulta las relaciones interpersonales, interfiere en su función ejecutiva y distorsiona el proceso de toma de decisiones orientado a metas de aprendizaje formales.

Respecto de las percepciones en torno al proceso de enseñanza aprendizaje de los estudiantes entrevistados, es posible constatar la figura del profesor/a como relevante en la función de establecer andamiajes cognitivos entre su cultura y la escolar formal. Desde esta perspectiva y de manera compartida tanto por estudiantes que se relacionan de manera 
complementaria como contradictoria con la escuela, el profesor con el que acceden a aprendizajes de significancia se caracteriza por establecer una interacción cálida, empática y provista de un diálogo constante, que incorpora sus inquietudes, necesidades e interpretaciones verbalizadas desde su cultura de origen respecto de la cultura escolar formal que le propone el profesor como situación de aprendizaje en el aula. En este sentido, los relatos muestran que es posible construir puentes que desde un punto dialéctico permitan superar la contradicción. Esto ocurre cuando el profesor es reconocido por sus alumnos como respetuoso y afectuoso con ellos, lo que actualmente se sintetiza como la aceptación del otro como el legítimo otro (Zapata et al., 2013). A este respecto, también se puede consignar que las escuelas y los profesores que dan una alta prioridad a la formación personal de los estudiantes, son los que pueden obtener importantes logros en términos del aprendizaje. No obstante, este logro requiere una gestión pedagógica y curricular altamente especializada (Bellei et al., 2014).

Una de las limitaciones del presente trabajo se refiere al estudio de los proyectos de vida del estudiantado en el mediano plazo que no pudieron ser contrastados al momento del desarrollo de la investigación, debido a las particularidades de las escuelas en estudio, las cuales finalizan el ciclo de enseñanza en la educación primaria. En este contexto, surge la posibilidad de proyectar esta línea de investigación, explorando las trayectorias escolares de los sujetos en educación secundaria y su relación en la construcción de sus proyectos de vida.

\section{Referencias}

Bellei, C., Valenzuela, J., Vanni, X., \& Contreras, D. (2014). Lo aprendí en la escuela. ¿Cómo se logran procesos de mejoramiento escolar? Santiago: LOM.

Bernstein, B. (1990). Clases, códigos y control: la estructura del discurso pedagógico. Madrid: Morata.
Bernstein, B. (1977). Class, codes and control, Vol. 3, Towards a theory of educational transmissions. Londrés: Routledge and Kegan Paul.

Bertaux, D. (1999). El enfoque metodológico: su validez metodológica, sus potencialidades. Proposiciones, 29. Recuperado de http://ww w.sitiosur.cl/r.php?id=436

Bourdieu, P., \& Passeron, J. (1977). La reproducción. Elementos para una teoría del sistema de enseñanza. Barcelona: Laia.

Bourdieu, P. (1997). Capital cultural, escuela y espacio social (1.a ed., I. Jiménez Comp. y Trad.). Buenos Aires: Siglo XXI.

Brandi, L., Filippa, N., \& Schiattino, E. (septiembre, 2009). Desplazamientos, reconversiones y desgarros en las trayectorias familiares de los alumnos de sectores populares. Trabajo presentado en el $\mathrm{X}$ Congreso Nacional de Investigación Educativa, Veracruz, México. Recuperado de http://www.comie.org.mx/congreso/me moriaelectronica/v10/pdf/area_tematica_1 4/ponencias/1293-F.pdf

Castro, A., Díaz, A., Ortiz, B., Figueroa, C., Maldonado, C., Muñoz, D., ... V. Yáñez. (2009). Dialéctica de los aprendizajes y ruptura del círculo de bajos aprendizajes en sectores de alta vulnerabilidad [Proyecto Conicyt, Soc-15]. Santiago de Chile: Conicyt. Recuperado de http://www2.udec .cl/panorama/imasd/n18/p05.htm

Castro, A., Figueroa, B., \& Maldonado, C. (2012). Estrategias de alfabetización funcional en contexto intercultural $y$ vulnerabilidad social. Concepción, $\mathrm{CH}$ : Editorial Universidad de Concepción.

Cornejo, M. (2006). El enfoque biográfico: trayectorias, desarrollos teóricos y perspectivas. Psykhe, 15(1), 95-106. Recuperado de http://www.psykhe.cl/index .php/psykhe/article/view/326

Cornejo, M., Mendoza, F., \& Rojas, R. (2008). La investigación con relatos de vida: pistas y opciones del diseño metodológico. Psykhe, 17(1), 29-39. Recuperado de http://www.psykhe.cl/index .php/psykhe/article/view/189/186 
Lainé, A. (1998). Faire de sa vie une histoire. Theories et ratiques de l'histoire de vie en formation. Paris: Desclée de Brouwer.

Willis, P. (1988). Aprendiendo a trabajar. Cómo los chicos de la clase obrera consiguen trabajo de clase obrera. Madrid: Akal.

Zapata, P., Castro, A., \& Rodríguez, G. (2013). Estrategias usadas por los estudiantes para superar el círculo de la reproducción social. REXE, 12(23), 89-102.

\section{Notas}

1 Se incluirán citas textuales extraídas de los relatos de vida de los participantes. Los nombres de los estudiantes son ficticios y cada escuela incluida en el estudio se ha designado con una letra.

* Artículo de investigación. Este artículo se desarrolló en el contexto del Proyecto Anillos CONICYT SOC - 15, titulado "Dialéctica de los aprendizajes y ruptura de los círculos de bajos aprendizajes". 